\title{
Thyroid Stimulating Hormone Increased, CTCAE
}

National Cancer Institute

\section{Source}

National Cancer Institute. Thyroid Stimulating Hormone Increased, CT CAE. NCI

Thesaurus. Code C143220.

A disorder characterized by an increase in thyroid stimulating hormone. 